J. Clin. Chem. Clin. Biochem.

Vol. 19, 1981, pp. $485-490$

\title{
Zum Stoffwechsel des Doxylamin: Isolierung, Identifizierung und Synthese einiger Metabolite
}

\author{
Von W. Gielsdorf \\ Direktion Polizeitechnische Untersuchungen, Berlin und \\ K. Schubert
}

Institut für org. Chemie der Freien Universität Berlin

(Eingegangen am 5. Januar/2. März 1981)

Zusammenfassung: Nach Einnahme therapeutischer Dosen von Doxylamin konnten im menschlichen Harn mit Hilfe chromatographischer und spektroskopischer Methoden - neben der unveränderten Ausgangsbindung (I) - fünf weitere Ausscheidungsprodukte nachgewiesen werden, deren chemische Strukturen diskutiert und z.T. durch Synthesen bewiesen wurden.

Wie die vorliegenden Ergebnisse zeigen, sind bei der Biotransformation des Doxylamins beim Menschen folgende Abbauwege zu erkennen: Neben den sukzessiven Desalkylierungen am Stickstoff, die über das N-Desmethyl-doxylamin(II) zum $\mathrm{N}$,N-Didesmethyl-doxylamin(III) führen, sind dies Spaltungen an der Benzhydrylether-Gruppe, die zu den Abbauprodukten 1-Phenyl-1-(2-pyridyl)-ethanol(V), 1-Phenyl-1-(2-pyridyl)-ethan(VI) und 1-Phenyl-1-(2-pyridyl)-ethen(VII) führen. Für VI und VII muß jedoch eine Artefaktbildung bedacht werden.

Für ein weiteres Abbauprodukt (IV) war eine eindeutige Strukturzuordnung aufgrund der geringen Substanzmenge nicht möglich.

Das analytische Verhalten (Hydrolyse!) der Reinsubstanz und freien Base wird ebenso eingehend diskutiert wie die Rolle der chemischen Ionisations-Massenspektrometrie mit verschiedenen Reaktandgasen bei der Untersuchung biologischer Extrakte.

\section{Biotransformation of doxylamine: Isolation, identification and synthesis of some metabolites}

Summary: After administration of therapeutic doses of doxylamine, the unchanged drug(I) and five degradation products were detected in human urine; their chemical structures are discussed and - to some extent - confirmed by synthesis. The results show that biotransformation of doxylamine in man takes place by the following routes: successive dealkylations at the nitrogen atom, giving N-demethyl-doxylamine(II) and N.N-didemethyl-doxylamine(II); cleavage at the benzhydrylether-function, resulting in the formation of 1-phenyl-1-(2-pyridyl)-ethanol(V), 1-phenyl1-(2-pyridyl)-ethane(VI) and 1-phenyl-1-(2-pyridyl)-ethene(VII). VI and VII may be artefacts.

Identification of an additional degradation product $(=I V)$ was not possible, because the isolated quantities were too small.

The analytical properties (hydrolysis!) of the pure substance and free base are thoroughly discussed, as well as the role of Chemical İonization Mass Spectrometry with various reagent gases for the examination of biological extracts.

\section{Einfithrung}

Doxylamin (Phenyl-2-pyridylmethyl-B=N;N-dimethylaminoethylether) findet hauptsächlich als Antihistaminicum in zahlreichen Arzneimitttelspezialitäten mediziniṣche Verwendung: so in Präparaten zur Bekämpfung von Erkrankungen der Atmungsorgane (1-6), von
Migräne und Allergien (7-8), zur Beruhigung (9) bzw. Bekämpfung der Reisekrankheit und des Schwangerschaftserbrechens.

Als ein Bestandteil mehrerer in der letzten Zeit in das Kreuzfeuer der Kritik geratener Präparate $(10,11)$, wie Bendectin (Lenotan ${ }^{\circledR}$ ), ist die Verbindung von aktuellem 
Interesse (12-20). Unser Interesse jedoch wurde bei der Untersuchung einer akzidentellen Intoxikation geweckt, als wir feststellen mußten, daß über das Biotransformationsverhalten der Verbindung im menschlichen Organismus keine Angaben zu finden waren (21), obwohl Intoxikationen (bei Kindern) mit tödlichem Ausgang in der Literatur beschrieben sind $(22,23)$.

Die bei l.c. (21) zitierte Arbeit von Snyder et al. (24) befaßt sich ausschließlich mit Metabolismus-Untersuchungen bei Hund und Ratte und stammt aus dem Jahre 1948: Metaboliten konnten nicht identifiziert werden.

Im Verlaufe der vorliegenden Untersuchungen konnten neben der unveränderten Ausgangsverbindung im menschlichen Harn eine Anzahl Ausscheidungsprodukte nachgewiesen und identifiziert werden, deren chemische Strukturen teilweise durch Synthesen bewiesen wurden.

Von besonderem Interesse erscheint das analyțische Verhalten der Reinsubstanz bzw. der freien Base: diese zersetzt sich zu Verbindungen, die auch als Metaboliten im Harn nach Doxylamin-Einnahme nachgewiesen werden konnten! Dies gilt auch für die Reaktionsprodukte der sauren Hydrolyse mit Salzsäure.

\section{Methodik}

Ein gesunder freiwilliger männlicher Proband nahm eine Woche lang dreimal täglich eine Kapsel Doxylamin (entspr. $75 \mathrm{mg} / \mathrm{d}$ ) ein.

Der Urin wurde bis einschließlich zwei Tage nach der letzten Einnahme in Braungasflaschen gesammelt und bis zur Aufbereitung kühl gelagert (keine Zusätze).

Vor Versuchsbeginn wurden etwa 1,51 Harn als Leerprobe gesammelt und wie die medikamenthaltigen Harne behandelt.

Während des Versuchs wurde die gewohnte Ernährung beibehalten, jedoch auf Kaffee, Tee und Konservengerichte ebenso verzichtet wie auf andere Medikamente, etwa Vitaminpräparate.

\section{Extraktion des Harnes}

Der Harn (insgesamt etwa $4 \mathrm{l}$ ) wurde jeweils vor und nach salzsaurer Hydrolyse bei pH 2-3 und 10-12 mit Chloroform extrahiert. Zur salzsauren Hydrolyse wurden jeweils drei Teile Urin mit einem Teil konz. Salzsäure versetzt und eine Stunde unter Rückfluß gekocht.

Zur enzymatischen Spaltung der Konjugate wurden $50 \mathrm{ml}$ Harn mit Acetatpuffer auf pH 5,5 eingestellt, mit $2 \mathrm{ml} \beta$-Glucuronidase/ Arylsulfatase (Merck) versetzt und im Trockenschrank bei $37^{\circ} \mathrm{C}$ über Nacht gehalten. Die Extraktion erfolgte bei pH 3-3,5 und 9-10 mit einem Gemisch aus Chloroform und Isopropanol $(3+1)$ je dreimal.

Die so gewonnenen Extrakte wurden dann über Natriumsulfat getrocknet, filtriert und in vacuo bei $40^{\circ} \mathrm{C}$ eingedampft, wobei den basischen Fraktionen vorher ein Tropfen konz. Salzsäure zugesetzt wurde.

\section{Hydrolyseversuche mit der Reinsubstanz}

Methode 1: $50 \mathrm{mg}$ Substanz, $30 \mathrm{ml}$ Wasser und $30 \mathrm{ml} \mathrm{konz}$. Salzsäure wurden in einem Erlenmeyerkolben auf einem Asbestnetz.über der Bunsenflamme 6 min gekocht. Dann wurde bei
pH 2-3 und 10 mit Chloroform extrahiert. Die Extraktionsrückstände wurden in wenig Methanol aufgenommen und direkt chromatographiert (DC; GC/MS). Das geschilderte Extraktionsverfahren war bei allen getesteten Methoden gleich.

Methode 2: $50 \mathrm{mg}$ Substanz, $30 \mathrm{ml}$ Wasser und $30 \mathrm{ml} 250 \mathrm{~g} / \mathrm{kg}$ Salzsäure wurden $30 \mathrm{~min}$ im kochenden Wasserbad erhitzt.

Methode 3: $50 \mathrm{mg}$ Substanz, $30 \mathrm{ml}$ Wasser und $30 \mathrm{ml} 250 \mathrm{~g} / \mathrm{kg}$ Salzsäure wurden für 15 min unter Rückfluß gekocht.

Methode 4: wie Methode 3, aber 30 min refluxt.

\section{Gewinnung der freien Base}

Zur Gewinnung der freien Base wurde eine wäßrige Lösung von Doxylaminsuccinat mit Natronlauge alkalisch gestellt (etwa pH 9-9,5) und mit Chloroform extrahiert. Die organische Phase wurde über Natriumsulfat getrocknet, filtriert und nach Zusatz eines Tropfens konz. Salzsäure in vacuo eingedampft.

Es resultiert eine ölige Flüssigkeit, aus der sich nach kurzer Zeit Kristalle abscheiden.

\section{Dünnschichtchromatographie}

Verwendet wurden Fertigplatten $\mathrm{HF}_{254}$ (Merck). Bei der präparativen Schichtchromatographie wurden die zu isolierenden Substanzen durch mehrmaliges Rechromatographieren abgetrennt, die substanzhaltigen Zonen abgeschabt, mit warmem Methanol extrahiert, das Kieselgel abzentrifugiert und die organische Phase in vacuo eing eengt.

Drei $\mu$ l des Rückstandes wurden - gelöst in wenig Methanol direkt chromatographiert.

Als Fließmittel dienten (ml; Ammoniak $250 \mathrm{~g} / \mathrm{kg}$ ):

$\begin{array}{lr}\text { A Benzol/Dioxan/Ammoniak (Oberphase) } & 60+35+5 \\ \text { B Essigester/Methanol/Ammoniak } & 85+10+5 \\ \text { C Chloroform/Ethanol/Ammoniak (Unterphase) } & 80+15+5 \\ \text { D Chloroform/n-Heptan/Methanol } & 85+10+5 \\ \text { E Toluol/Ethanol/Ammoniak } & 80+20+1 \\ \text { F Chloroform/Methanol/Ammoniak } & 80+20+1 \\ \text { G Methanol/Ammoniak } & 100+1.5\end{array}$

Fließmittel $\mathrm{E}$ erwies sich am geeignetsten.

Zur Detektion wurden benutzt (34):

1 Kaliumiodoplatinat.

2 Dragendorff-Reagenz nach Thies \& Reuther und Nachbesprühen mit einer wäßrigen, $50 \mathrm{~g} / \mathrm{l}$ Eisen(III)-chlorid-Lösung.

Die UV-Spektren wurden mit dem Zeiss DMR 10, die IR-Spektren mit dem PE-328 Spektrophotometer aufgenommen (1 mg Sub$\operatorname{stanz}+200 \mathrm{mg} \mathrm{KBr}$ ).

Die ${ }^{1} H$-NMR-Spektren wurden mit dem Varian XL-100 erhalten.

Die Bedingungen für die Kombination Gaschromatograph/Massenspektrometer sind bei 1.c. (27) und (29) beschrieben.

\section{Synthesen}

N-Mono-desmethyl-doxylamin (Metabolit II)

Die Verbindung wurde nach der von Fahr (35) zur Synthese des $\mathrm{N}$-Mono-desmcthyl-p-bromdiphenhydramins beschriebenen Methode dargestellt.

\section{N.N-Dide̊smethyl-doxylamin (Metabolit III)}

1-Phenyl-1-(2-pyridyl)-ethanol (Metabolit V) $(15 \mathrm{~g}), 40 \mathrm{ml}$ 2Chlorethanol und 1,5 ml konz. Salzsäure wurden nach $1 . c$. (35) umgesetzt. Das Reaktionsgemisch wurde anschließend mit $250 \mathrm{ml}$ Ethanol (mit $\mathrm{NH}_{3}$-Gas gesättigt) versetzt und eine Woche bei Raumintemperatur belassen. Nach Aufarbeitung (36) fiel das Amin als freie Base an. 
1-Phenyl-1-(2-pyridyl)-ethanol (Metabolit V)

Die Dảrstellung erfolgte nach der Methode von Sperber et al. (37).

1-Phenyl-1-(2-pyridyl)-ethan (Abbauprodukt VI)

Verbindung $\mathrm{V}$ wurde durch Kochen mit Thionylchlorid in das entsprechende Chlorid überführt, das dann mit Zink/Eisessig reduziert wurde.

\section{Ergebnisse und Diskussion}

Nach Einnahme von Doxylamin konnten im alkalischen Extrakt vor Hydrolyse mit Hilfe spektroskopischer und chromatographischer Methoden fünf Ausscheidungsprodukte nachgewiesen werden:

Neben der unveränderten Ausgangsverbindung (I) wurden noch deren Mono- bzw. Didesmethyl-Derivate (II, III) als Metaboliten identifiziert.

Unter den Bedingungen der Elektronenstoß-Ionisation (EI) liefern diese Verbindungen Massenspektren, die durch die $\alpha$-Spaltprodukie m/e 58, 44 bzw. 30 und wenig intensive Pseudomolekülionen bei $\mathrm{m} / \mathrm{e}$ 271, 257 und 243 gekennzeichnet sind; ein für diese Verbindungsklasse typisches Verhalten (Abb. 1 und 2).

Daher gelangte das Verfahren der chemischen Ionisation (CI) zur Anwendung, da dieses - bedingt durch die "weicheren" Ionisationsbedingungen - eine eindeutige Molekulargewichtszuordnung, durch die Erhöhung der Selektivität ${ }^{1}$ ) eine Steigerung von Empfindlichkeit und Präzision und da im höheren und damit störungsfreierem Massenbereich gemessen wird, eine Reduzierung spektraler Überschneidungen garantiert $(26,27)$.

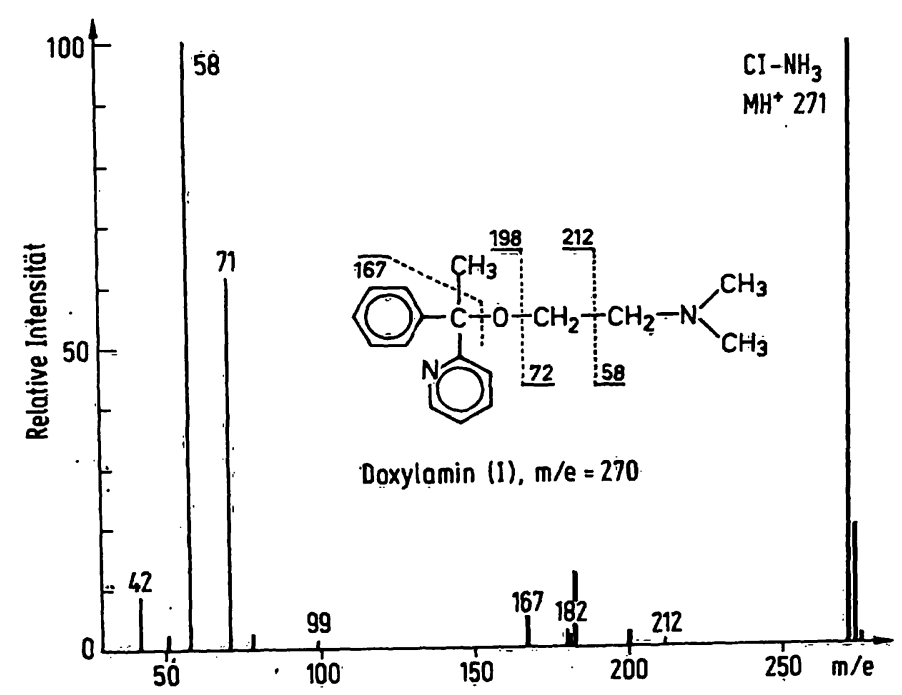

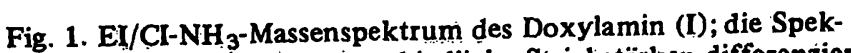
tren sind durch unterschiedliche Strichstärken differenziert.

\footnotetext{
1) Eine eingehende Diskussion des Begriffs ,Selektivität" findet sich bei l.c. (25).
}

J. Clin. Chem. Clin. Biochem. / Vol 19, 1981 / No. 7

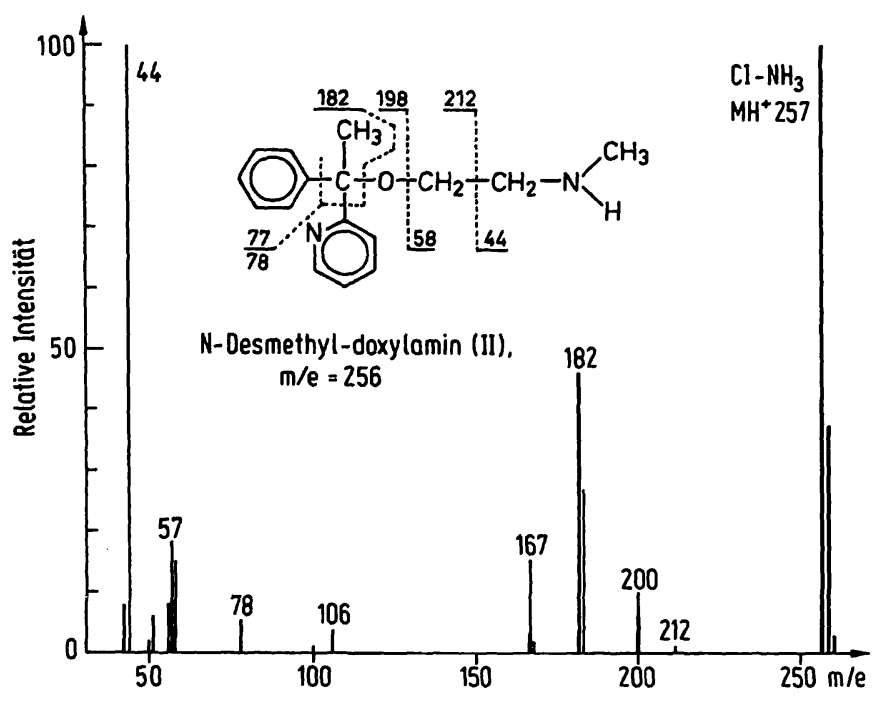

Fig. 2. EI/CI-NH 3 -Massenspektrum des Hauptmetaboliten NMono-desmethyl-doxylamin (II); die Spektren sind durch unterschiedliche Strichstärken differenziert.

Zur Untersuchung der basischen Extrakte wurde Ammoniak als selektives (!) Reaktandgas benutzt (28-30), für die bei saurer Reaktion extrahierten Proben wurden zusätzlich iso-Butan bzw. Methan verwendet.

Die UV-Spektren von I-III zeigten in Methanol Absorptionsmaxima bei $260 \mathrm{~nm}$ (Schulter bei 256 und $265 \mathrm{~nm}$ ).

Das IR-Spektrum von Verbindung II zeigt Abbildung 3: charakteristische Banden liegen bei $306 \mathrm{C} \mathrm{cm}^{-1}$ (C-H aromat.), 1610,1585 und $1490 \mathrm{~cm}^{-1}$ ( $C=C$ aromat.), 2980 und $2930 \mathrm{~cm}^{-1}$ (C-H methyl) und 1160 und $1085 \mathrm{~cm}^{-1}$ (C-O).

Da Metabolit III unter den angegebenen Bedingungen nicht unzersetzt gaschromatographierbar ist, wurde ein Teil der Probe mit N-Methyl-N-trimethylsilyl-trifluoracetamid (31) während $60 \mathrm{~min}$ bei $80^{\circ} \mathrm{C}$ im Alu-Block silyliert. Das CI-Massenspektrum der Reaktionslösung zeigt, daß die Verbindung N-bistrimethylsilyliert wird, wie der base-peak bei $\mathrm{m} / \mathrm{e} 372(=\mathrm{m} / \mathrm{e} 243+2 \times$ trimethylsilyl -15) beweist.

Im Fließmittel E ergaben sich für die mit Kaliumiodoplateat braunrot und mit Dragendorff-Reagenz orange anfärbbaren Verbindungen $R_{F}$-Werte von $0,44,0,31$ und 0,78 .

Für ein weiteres, im Harn nachweisbares Abbauprodukt (IV) konnte aufgrund der äußerst geringen Substanzmenge ein Strukturbeweis nicht erbracht werden:

Das ET-Massenspektrum (Tab. 1) zeigt als Ion mit der höchsten Masse m/e 198, als base-peak m/e 86 und weitere intensive Ionen bei $\mathrm{m} / \mathrm{e} 167,152,78$ und 58; die CI-Spektren ein Quasimolekükion bei $\mathrm{m} / \mathrm{e} 285 \mathrm{mit}$ $\simeq 50 \%$ rel. Int., einen base-peak bei $\mathrm{m} / \mathrm{e} 181$ und starke Ionen bei $\mathrm{m} / \mathrm{e} 104$ und 85 .

Metabolit V (= 1-Phenyl-1-(2-pyridyl)-ethanol) wurde nur im basischen Extrakt nach Hydrolyse beobachtet; 


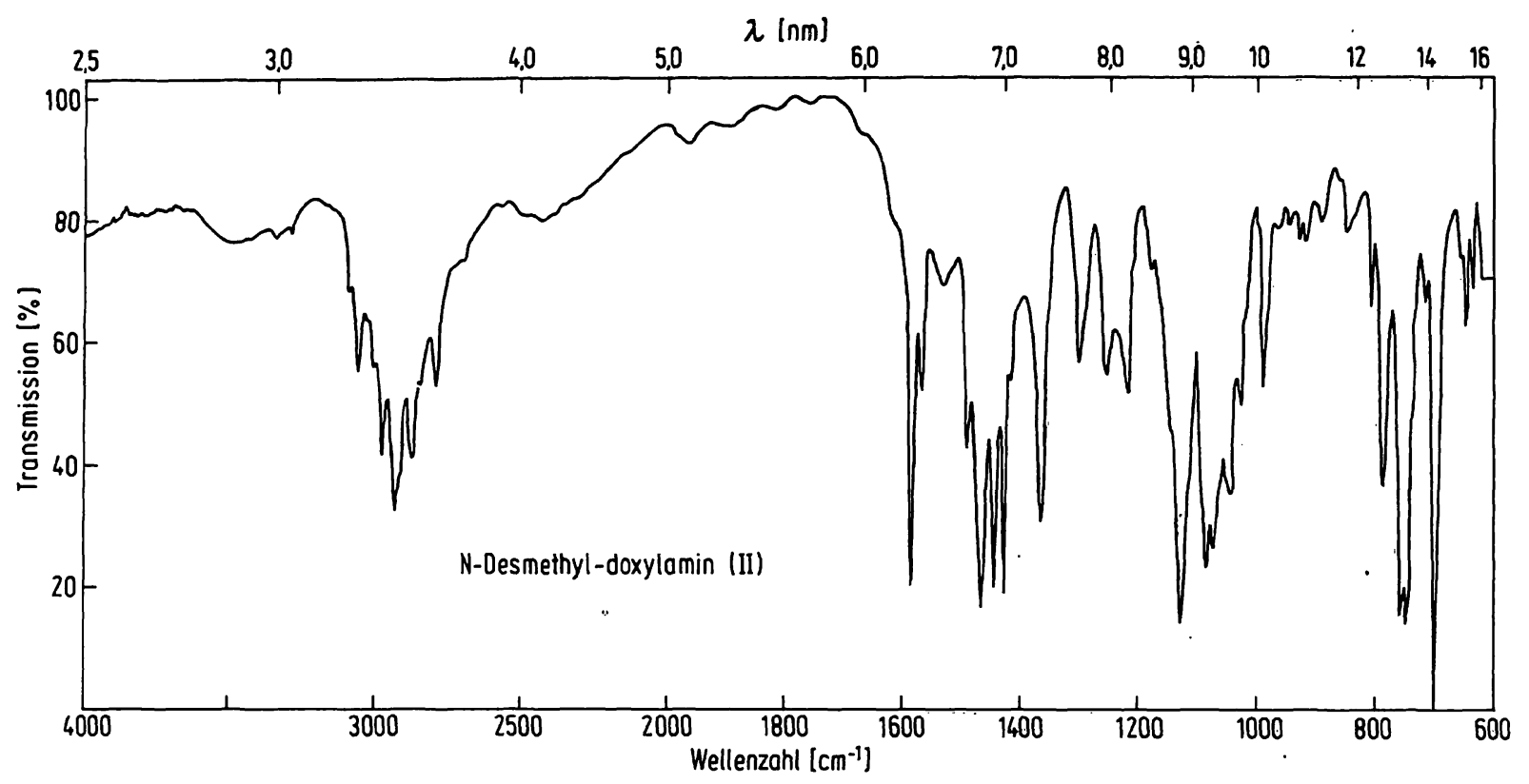

Fig. 3. IR-Spektrum von II.

Tab. 1. Die massenspektrometrischen Kenndaten (EI/CI) der nachgewiesenen Verbindungen.

\begin{tabular}{|c|c|c|c|c|}
\hline Verbindung & $\begin{array}{l}M_{\mathrm{r}} \\
\text { Summen- } \\
\text { formel }\end{array}$ & $\begin{array}{l}\text { EI } \\
\text { Molekülion; starke Ionen } \\
\text { (m/e;\% rel. Int.) }\end{array}$ & Reaktandgas & $\begin{array}{l}C I \\
\text { Quasimolekülion; starke Ionen } \\
\text { (m/e;\% rel. Int.) }\end{array}$ \\
\hline III & $\begin{array}{l}242 \\
\mathrm{C}_{15} \mathrm{H}_{18} \mathrm{~N}_{2} \mathrm{O}\end{array}$ & $\begin{array}{l}\left.\text { Bis-trimethylsilyl-Derivat (Cl-NH } \mathrm{NH}_{3}\right) \\
405(8) ; 372(100) ; 184(3)\end{array}$ & $\begin{array}{l}\mathrm{NH}_{3} \\
\text { iso-Butan }\end{array}$ & $\begin{array}{l}243(10) ; 200(15) ; 184(100) ; 155(2) \\
243(40) ; 171(5) ; 114(100) ; 85(79)\end{array}$ \\
\hline IV & $\mathrm{C}_{17}^{284} \mathrm{H}_{20} \mathrm{~N}_{2} \mathrm{O}_{2}$ & $\begin{array}{l}198(18) ; 182(62) ; 167(50) ; \\
152(4) ; 135(6) ; 86(100) ; \\
78(24) ; 72(17) ; 58(36)\end{array}$ & $\begin{array}{l}\mathrm{NH}_{3} \\
\text { iso-Butan }\end{array}$ & $\begin{array}{l}285(78) ; 184(100) ; 104(82) \\
285(100) ; 180(48) ; 104(70)\end{array}$ \\
\hline v & $\begin{array}{l}199 \\
\mathrm{C}_{13} \mathrm{H}_{13} \mathrm{NO}\end{array}$ & $\begin{array}{l}199(48) ; 182(37) ; 167(3) ; \\
156(10) ; 122(27) ; 105(18) ; \\
79(58) ; 51(34) ; 43(100)\end{array}$ & $\begin{array}{l}\mathrm{NH}_{3} \\
\text { iso-Butan }\end{array}$ & $\begin{array}{l}200(70) ; 184(60) ; 182(100) ; 122(38) \\
200(100) ; 182(70) ; 167(2)\end{array}$ \\
\hline VI & $\begin{array}{l}183 \\
\mathrm{C}_{13} \mathrm{H}_{13} \mathrm{~N}\end{array}$ & $\begin{array}{l}183(42) ; 182(100) ; 167(54) ; \\
106(24) ; 77(38) ; 51(42)\end{array}$ & $\begin{array}{l}\mathrm{NH}_{3} \\
\text { Methan }\end{array}$ & $\begin{array}{l}184(100) ; 108(4) \\
184(100) ; 212(20) ; 106(5)\end{array}$ \\
\hline VII & $\begin{array}{l}181 \\
\mathrm{C}_{13} \mathrm{H}_{11} \mathrm{~N}\end{array}$ & $\begin{array}{l}181(24) ; 180(100) ; 152(8) \\
103(3) ; 77(15) ; 63(2) ; 51(20)\end{array}$ & $\begin{array}{l}\mathrm{NH}_{3} \\
\text { Methan }\end{array}$ & $\begin{array}{l}182(100) ; 108(6) \\
182(100) ; 210(15) ; 167(3) ; 106 \text { u. } 79(2)\end{array}$ \\
\hline
\end{tabular}

durch hochauflösende Massenspektroskopie wurde die Summenformel zu $\mathrm{C}_{13} \mathrm{H}_{13} \mathrm{NO}$ bestimmt. Durch Abspaltung von $\mathrm{OH}$ entsteht daraus das Ion $\mathrm{m} / \mathrm{e} 182$ $\left(\mathrm{C}_{13} \mathrm{H}_{12} \mathrm{~N}\right)$, der Verlust von $\mathrm{CH}_{3}$ führt zu m/e 167 $\left(\mathrm{C}_{12} \mathrm{H}_{9} \mathrm{~N}\right)$; als wichtige Primär-Fragmentierung tritt noch die Abspaltung des Pyridyl-Restes auf, die zu den Fragmenten m/e 78 bzw. 122 führt.

Im IR-Spektrum (Film) liegen die charakteristischen Banden bei $3400 \mathrm{~cm}^{-1}$ (breit; OH), $3060 \mathrm{~cm}^{-1}$ (C-H aromat.), $1660 \mathrm{~cm}^{-1}(C=N), 1590$ und $1490 \mathrm{~cm}^{-1}$ $\left(\mathrm{C}=\mathrm{C}\right.$ aromat.) und 1098 und $1130 \mathrm{~cm}^{-1}$ (C-O).

Der $R_{F}$ Wert im Fließmittel E betrug 0,64; mit den angegebenen Detektionsmitteln zeigte $\mathrm{V}$ eine blauviolette bzw. orange Anfärbung.
Durch hochauflösende Massenspektrometrie wurde die Elementarzusammensetzung der Abbauprodukte VI und VII zu $\mathrm{C}_{13} \mathrm{H}_{13} \mathrm{~N}$ bzw. $\mathrm{C}_{13} \mathrm{H}_{11} \mathrm{~N}$ bestimmt. Daraus und aus den in Tabelle 1 aufgeführten Massenspektren läßt sich für VI zwanglos die Struktur eines 1-Phenyl-1-(2pyridyl)-ethans, für VII die eines 1-Phenyl-1-(2-pyridyl)ethens ableiten.

Verbindung VII wurde außerdem in den sauren Extrakten vor und nach Hydrolyse und bei Zersetzung der freien Base sowie der salzsauren Hydrolyse nachgewiesen, nicht jedoch in den Fraktionen nach enzymatischer Spaltung: eine Artefakt-Bildung ist daher, wie auch für VI, nicht auszuschließen. Auf der DC-Platte läßt sich VI mit den beschriebenen Sprühmitteln blauviolett bzw. xotorange anfärbeñ $\left(\tilde{R}_{F}\right.$-Wert im Laufmittel E: 0,70$)$. 


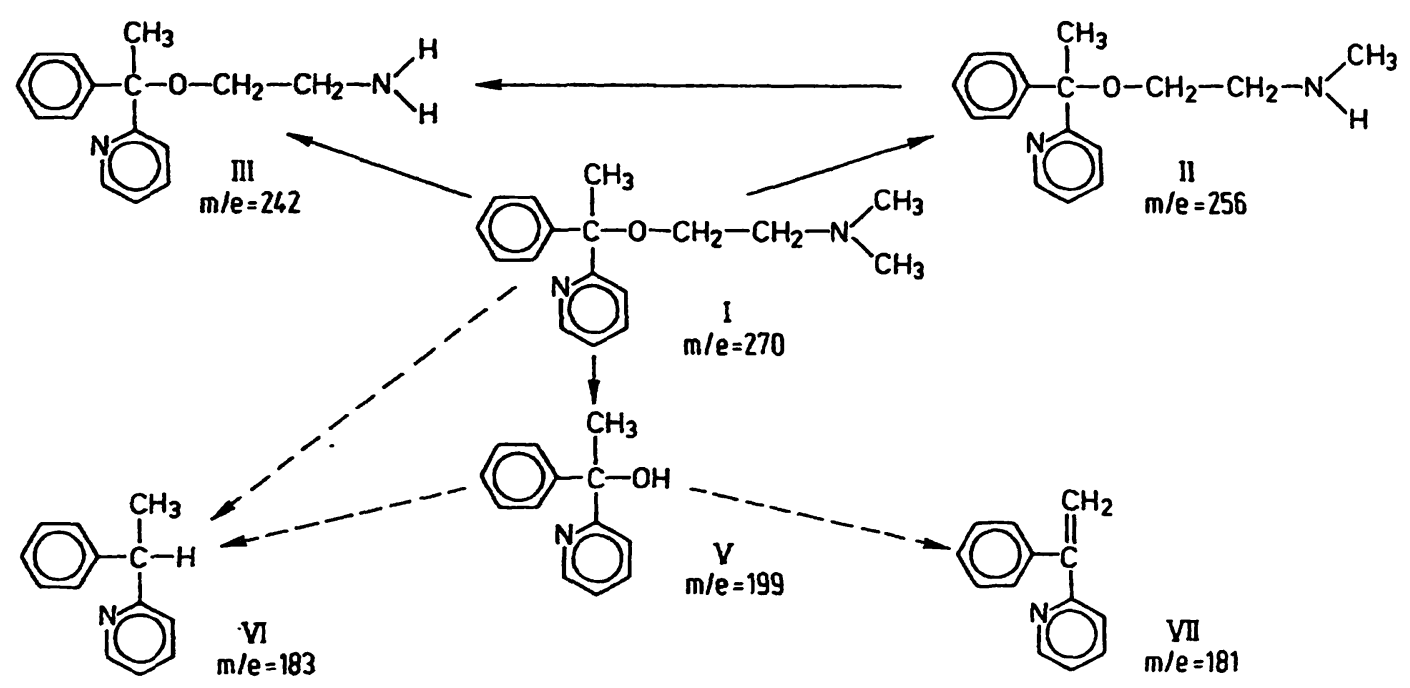

Fig. 4. Übersicht über die im menschlichen Harn, bei der sauren Hydrolyse und der Zersetzung der freien Base nachgewiesenen Abbauprodukte des Doxylamin.

Die in $\mathrm{CCl}_{4}$ aufgenommenen ${ }^{1} \mathrm{H}-\mathrm{NMR}$-Spektren der Verbindung II, $\mathrm{V}$ und VI zeigen bei $\delta=7.0-8.6$ ein Multiplett für die aromatischen Protonen, die benzylische Methylgruppe ein Singulett bei $\delta=2.0$ (II), 1.9 (V) bzw. ein Quadruplett bei $\delta=1.65$ (VI). Bei II liegt das Triplett der $\mathrm{O}-\mathrm{CH}_{2}$-Gruppe bei $\delta=2.85$, das Multiplett der $\mathrm{CH}_{2}-\mathrm{N}$-Gruppe bei $\delta=3.48$ und das Dublett der $\mathrm{N}$ $\mathrm{CH}_{3}$-Gruppe bei $\delta=2.5$ (Angaben in ppm; Int. Standard Trimethylsilan).

\section{Das Hydrolyseverhalten der Reinsubstanz und das analy- tische Verhalten der freien Base}

Die in der Literatur für die Spaltung der Konjugate mit Mineralsäuren beschriebenen Methoden zeigen beträchtliche Unterschiede in den Reaktionsbedingungen, insbesondere in Bezug auf die Hydrolysedauer und die angegebenen Säurekonžentrationen. Die vier getesteten - in der toxikologischen Praxis gebräuchlichen - Hydrolysemethoden führten beim Doxylaminsuccinat alle zu den gleichen Reaktionsprodukten, wobei die quantitativen Verhältnisse die hohe Stabilität der Verbindung gègen Säuren widerspiegeln: mit Hilfe der DC und GC/MS wurden in den Reaktionsansätzen jeweils $87 \%$ unveränderte Ausgangsverbindung (I), 9\% (V) und 4\% (VII) nachgewiesen; ein weiteres denkbares Hydrolyseprodukt, das 2-Benzoylpyridin, konnte dagegen nicht beobachtet werden.

Die nach der im Teil II (Methodik) beschriebenen Methode gewonnene freie Base zersetzt sich sofort: bei deren DC zeigten sich eine ganze Reihe mit den angegebenen Detektionsmitteln anfärbbarer Substanzflecke, von denen mehrere identifiziert werden konnten (GC/MS):

neben der Ausgangsverbindung (ca. 85\%) gelang noch der Nachweis der Verbindungen V (2\%), VI (4\%) und
VII (6\%). Diese Besonderheiten im chemischen Verhalten sind bei der Bereitung von Referenzlösungen - z. B. bei der DC und GC - zu berücksichtigen; andererseits jedoch bietet das charakteristische Muster dieser Zersetzungsprodukte auf der DC-Platte einen zusätzlichen Identifizierungshinweis! Erwähnenswert erscheint noch die Tatsache, daß die sich sofort nach Herstellung der freien Base bildenden Zersetzungsprodukte über Monate hinweg stabil (,,im Gleichgewicht") bleiben, die Lösung also keinerlei qualitative oder quantitative Veränderungen mehr erfährt.

\section{Schlußfolgerungen}

Aufgrund der vorliegenden Ergebnisse lassen sich für die Biotransformation von Doxylamin beim Menschen mehrere Abbauwege aufzeigen:

Sukzessive Demethylierung am Stickstoffatom (II, III) führt zum entsprechenden Amin; derartige N-Desalkylierungen sind in der Literatur gut dokumentiert (Clofedanol, Diphenhydramin, Isoaminil (28), Clobazam (27), Dihydrocodein (26), Mefexamid (30)).

Die zweite Gruppe von Abbauprodukten (V-VII) wird bei den Spaltungen an der Benzhydrylether-Funktion gebildet, ein Abbauweg, der für zahlreiche KolaminDerivate (u.a. Diphenylhydramin, Bromazin, Brompheniramin) dokumentiert ist $(21,35)$.

Das Ausscheidungsverhalten der $\alpha$-Methyl-Derivate des Kolamins dagegen ist nur sehr lückenhaft in der Literatur beschrieben; als Beispiele seien Mephenhydramin (Moxast ${ }^{\circledR}$ ), Chlorphenoxamin (Systral ${ }^{\circledR}$ ), Embramin (Mebryl ${ }^{\circledR}$ ) und nicht zuletzt das Doxylamin angefuihrt (21). 
Die Produkte einer oxidativen Desaminierung, Ringhydroxylierungen oder $\mathrm{N} \rightarrow \mathrm{O}$ Bildung konnten nicht nachgewiesen werden.

Die quantitativen Untersuchungen ergaben, daß über die Hälfte (etwa 60\%) der eingenommenen Doxylaminmenge wieder unverändert mit dem Ham ausgeschieden wird: daher lagen wohl auch die nachgewiesenen Abbauprodukte - bis auf II - in relativ geringen Konzentrationen vor.

Der Nachweis einer Doxylamin-Einnahme wird daher zweckmäßigerweise über die in hoher Konzentration im alkalischen Urinextrakt vorliegende Ausgangsverbindung geführt; das charakteristische Muster der Zersetzungsprodukte auf der DC-Platte ergibt dabei einen zusätzlichen Hinweis.
Eine papierchromatographische Nachweismethode für I beschreiben Belles \& Sievert (32); neben einigen analytischen Daten sind bei l.c. (33) und (34) noch Angaben zur Pharmakologie referiert.

\section{Danksagung}

Wir sind Herrn Dr. G. Eckhardt, Institut für org. Chemie und Biochemie der Universität Bonn, für die Aufnahme der hochaufgelösten Massenspektren sowie den Firmen Chephasaar, St. Ingbert und Merrell Pharma, Groß-Gerau für die Überlassung von Reinsubstanz und Literatur zu Dank verpflichtet.

\section{Literatur}

1. Aviado, D. M., Bianchi, A. \& Drimal, J. (1976), Arch. Int. Pharmacodyn. Ther. 216, 216-224.

2. Romizi, S., Amici, A., De Benedictis, F. M. \& Luciana, G. (1979), Gazz. Med. Ital. 138, 7-12.

3. Legler, F. \& Jansen, W. (1977), Arneimittel Forsch. 27, 883-888.

4. Edel, G. (1972), Med. Monatsschr. 26, 27-29.

5. Weinberg, E. G., Shore, S. C. \& Anderton, M. (1974), Curr. Med. Res. Opin. 2, 222-230.

6. Weinberg, E. G. \& Shore, S. C. (1975), Curr. Med. Res. Opin. $2,264-269$.

7. Sommerville, B. W. (1977), Med. J. Austr. 1, 865-866.

8. Kagan. G. \& Masheter, H. C. (1978), Curr. Med. Res. Opin. $5 / 9,709-713$.

9. Beipackzettel/Mitt. der Firmen Chephasaar, St. Ingbert (Doxylamin) und Merrell Pharma, Groß-Gerau (Mereprine ${ }^{\circledR}$ ).

10. Dt. Apoth. Ztg. (1980), 120, 714.

11. Der Stern (1979), 43, 222.

12. Shapiro, S., Heinonen, O. P., Siskind, V., Kaufman, D. W. Monson, R. R. \& Slone, D. (1977), Am. J. Obstet. Gynecol. $128,480-485$.

13. Check, W. A. (1979), J. Amer. Med. Ass. 242, 2518

14. Smithells, R. W. \& Sheppard, S. (1978), Teratology 17, $31-35$

15. Patterson, D. C. (1977), Can. Med. Assoc. J. 116, 1348.

16. Miklovich, L. \& van den Berg, B. J. (1976), Am. J. Obstet. Gynecol. 125, 244-248.

17. Donnai, D. \& Harris, R. (1978), Br. Med. J. 1, 691-692.

18. Wheatley, D. (1977), Br. J. Obstet. Gynaecol. 84, 444-447.

19. Anonym (1978), Can. Med. Assoc. J. 117, 721-722.

20. Anonym (1978), Br. Med. J. 1, 1055-1056.
21. Pfeifer, S. (1980), Biotransformation von Arzneimitteln. Verlag Chemie, Weinheim.

22. Bayley, M., Walsh, F. M., Valaske, M. J. (1975), Clin. Pediatr. (Phila.) 14, 507-509.

23. Clarkson, S. G. \& Glanvill, A. P. (1977), Br. Med. J. 2, 459-460.

24. Snyder, F. H., Klahm, G. R. \& Werner, H. W. (1948), J. Am. Pharm. Assoc. 37, 420-423.

25. Fenselau, C. (1978), Selectivity in Biomedical Applications, in: High Performance Mass Spectrometry: Chemical Applications (Gross, M. L. ed.). ACS Series 70, 188-208, Washington.

26. Gielsdorf, W. (1980), Fresenius Z. Anal. Chem. 301, 434-435.

27. Gielsdorf, W. \& Herper, M. (1980), Z. Rechtsmed. 85, 295-303.

28. Gielsdorf, W. \& Holz, H. (1980), Dt. Apoth. Ztg. 120, 1353-1355.

29. Gielsdorf, W. \& Toffel-Nadolny, P. (1981), J. Clin. Chem. Clin. Biochem. 19, 25-30.

30. Gielsdorf, W., Z. Rechtsmed. - zur Publ. eingereicht.

31. Donike, M. (1969), J. Chromatogr. 42, 103-104.

32. Belles, Q. C. \& Sievert, H. W. (1955), J. Lab. Clin. Med. 46, 628-640.

33. Marsh, D. F. (1948), W. Virginia Med. J. 44, 265-269.

34. Clarke, E. G. C. (1969), Isolation and Identification of Drugs. The Pharmaceutical Press, London.

35. Fahr, W. (1978), Diss. Univ. Bonn.

36. Speer, H. G. (1938), J. Org. Chem. 2, 139

37. Sperber, N., Papa, D., Schwenk, E. \& Sherlock, M. (1949), J. Am. Chem. Soc. 71, 887-890.

Dr. rer. nat. Werner Gielsdorf c/o Direktion Polizeitechnische Untersuchungen Gothaer Straße 19

D-1000 Berlin 62 